\title{
Non alcoholic fatty liver disease is a predictor of subclinical Carotid Atherosclerosis in the presence of Metabolic Syndrome
}

\author{
Cemal Kemaloglu1, Melek Didem Kemaloglu² \\ ${ }^{1}$ Akdeniz University Medical Faculty, Department of Cardiovascular Surgery, Antalya/Turkey \\ ${ }^{2}$ Ataturk State Hospital, Department of Cardiology, Antalya/Turkey \\ Corresponding Author: Cemal Kemaloglu(orcid id: 0000-0002-3326-1990) \\ Akdeniz Üniversitesi Hastanesi, Kalp Damar Cerrahisi Poliklinigi. H Blok. 1.Kat. \\ ANTALYA/TÜRKIYE \\ Melek Didem Kemaloglu, Orcid ID: 0000-0002-3535-0401 \\ Email address: cemalkemaloglu@akdeniz.edu.tr
}

Cite this article as: Kemaloglu C, Kemaloglu M D. Non alcoholic fatty liver disease is a predictor of subclinical Carotid Atherosclerosis in the presence of Metabolic Syndrome. Nepalese Heart Journal 2019; Vol 16(1), 39-45

Received date: 1 st January 2019

Accepted date: 30th March 2019

\section{Abstract}

Objective: The aim of this study is to identify the relationship between carotid intima-media thickness (c-imt) and non-alcoholic fatty liver disease (NAFLD), and to determine whether NAFLD is an independent predictor for the progression of atherosclerosis.

Method: This is a prospective randomized controlled study. 103 NAFLD patients who have hepatosteatosis with grade II and above were enrolled in this study. Patients were divided into NAFLD with metabolic syndrome (MS) and NAFLD without MS groups and compared with 50 healthy people. Basal demographic characteristics and C-imt of all patients and control group were measured.

Results: C-imt and carotid cross sectional area rates in the NAFLD groups were significantly higher than those in the control group. The mean and max. c-imt levels were significantly higher in the NAFLD group with metabolic syndrome $(\mathrm{p}<0,001)$. Homeostatic Model of Assessment-Insulin Resistance (HOMA-IR) levels were increased in the group with metabolic syndrome than those in the group without metabolic syndrome, with statistical significance $(\mathrm{p}<0.001)$. There was no difference in c-imt levels between HOMA-IR positive and negative groups $(\mathrm{p}=0.254)$ in patients with NAFLD and without metabolic syndrome. There was only a mild positive corelation between c-imt levels and high sensitive C-Reactive protein (hs-CRP) levels in metabolic syndrome positive group ( $\mathrm{p}=0.026 \mathrm{r}=0.30$ ).

Conclusion: NAFLD was a significant predictor to determine the increased risk of carotid atherosclerosis.

Key words: atherosclerosis, carotid intima-media thickness, metabolic syndrome.

DOI: https://doi.org/10.3126/njh.v16i1.23898

\section{Introduction}

Non-alcoholic fatty liver disease (NAFLD), is a condition defined in increasing frequency in patients without excessive alcohol consumption characterized by significant lipid deposition in hepatocytes. The prevalence of NAFLD in the general population varies from $20 \%$ to $30 \%$ and it is the most common cause of elevated liver enzymes and chronic liver disease in the industrialized world. ${ }^{1}$

Previously, NAFLD was considered to be completely benign, which had rarely a clinical importance. But findings from recent studies suggest that non-alcoholic fatty liver disease is a metabolic disease in which insulin resistance is a common, primary factor in addition to hypertension, diabetes and atherosclerosis..$^{2-4}$ Abdominal obesity, type 2 diabetes, insulin resistance, hypertension, and dyslipidemia which are typical components of metabolic syndrome, are pathological conditions frequently associated with NAFLD and their association increases the possibility of the more complicated forms of NAFLD in patients. ${ }^{5,6}$ This situation strongly supports the idea that NAFLD may be a hepatic manifestation of metabolic syndrome (MS). Furthermore, recent publications showed that cardiovascular complications constitute the most common cause of death in NAFLD patients. ${ }^{?}$

Arterial wall thickness and stiffness, endothelial dysfunction, coronary artery calcification and many non-invasive markers can be utilized for early detection of subclinical atherosclerosis. A

@Nepalese Heart Journal. Nepalese Heart Journal retains copyright and work is simultaneously licensed under Creative Commons Attribution License CC - BY 4.0 that allows others to share the work with an acknowledgement of the work's authorship and initial publication in this journal. 
widely accepted, convenient marker of atherosclerosis is carotid artery intima-media thickness (c-imt) which is significantly associated with prevalence and incidence of carotid plaques. ${ }^{8,9}$ Non-invasive evaluation of c-imt by high-resolution B-mode ultrasonography has broad utility as a valuable end point for cardiovascular diseases. Carotid ultrasonography also is a method that is able to evaluate luminal diameter, intima-media thickness and presence or absence of plaques in carotid arteries in a safe and reproducible manner. Current recommendations suggest the use of carotid cross sectional area (CSA) measurements for the calculation of carotid artery stenosis however it is not yet validated in a large population. ${ }^{10} \mathrm{CSA}$ was calculated as the total artery area minus the lumen area assuming a circular lumen and an outer artery structure that was either circular or elliptical. The formula is: $\mathrm{A}=\pi \mathrm{r}^{2}-\pi(\mathrm{r}-\mathrm{IMT})^{2}$ where $\mathrm{A}$ is the arterial wall area, $r$ is the artery radius and IMT is wall thickness that is used to estimate wall area assuming circular configurations. ${ }^{11}$

In this study, we aimed to identify the relationship between c-imt and non-alcoholic fatty liver disease, and to determine whether NAFLD is an independent risk factor for the progression of atherosclerosis.

\section{Materials and Methods}

A total of 103 randomly selected patients who underwent hepatic ultrasonography by radiology in Akdeniz University, Antalya, Turkey between May 2010-May2011 and who had grade 2, 3, or 4 fatty liver disease were included in this study. Exclusion criteria were alcohol or hepatotoxic drug usage, viral or autoimmune hepatitis, previous history of coronary or peripheral artery disease. We diagnosed metabolic syndrome according to criteria using insulin resistance [according to World Heart Organization (WHO) 1999 MS diagnostic criterias $]^{12}$; and patients with NAFLD divided into two groups, NAFLD with metabolic syndrome $(n=55)$ and without metabolic syndrome $(\mathrm{n}=48)$. The control group included 50 healthy volunteers who were in same age and gender range.

Weight, height, waist circumference (WC) and hip circumference (HC) of patients were measured. Body Mass Index (BMI) and WC/HC ratios calculated. Levels of serum fasting glucose, alanine aminotransferase (ALT), aspartate aminotransferase (AST), gamma-glutamyl transferase(GGT), insulin, high sensitive C-Reactive protein(hs-CRP), glycated haemoglobin(HBA1c), triglyceride(TG), high density lipoprotein(HDL) and direct low density lipoprotein(dLDL) levels tested in morning blood after 12 hours of fasting. Homeostatic Model of Assessment-Insulin Resistance (HOMA IR) measured by using HOMA-IR $=\{$ Fasting insulin (mIU/L) $\mathrm{X}$ fasting blood glucose (mg/dl) X 0.9555$\} / 22.5$ formula. HOMAIR value was accepted as negative below 2.5 and positive over $2.5 . .^{13}$

We measured bilateral carotid intima-media thickness in all patients and in control group using high-resolution, carotid B-mode ultrasonography. We calculated carotid cross sectional area by the formula mentioned above for the study population. And we also evaluated the presence of plaques at carotid arteries.

Other factors, which could affect carotid atherosclerosis like dyslipidemia, diabetes, hs-CRP and HOMA-IR, investigated one by one in NAFLD patients with a dichotomic $(>0.9 \mathrm{~mm})$ result of c-imt. Linear correlation analyses were performed to identify the relationship between these factors and c-imt values.

The institutional Ethics Committee approved the study, and written informed consent for participation in the study was obtained from all individuals.

\section{Statistical analysis}

All the data was analysed with SPSS for Windows 13.0. Descriptive statistics of all the variables were given. The comparison of continuous variables on a group basis, KruskalWallis and one-way analysis of variance was used for analysis. Post-hoc test or bilateral comparisons were made to identify different groups. In the analysis of the categorical data chi-square test was used. Continuous variables were presented as mean $\pm S D$ and numbers (percent) are used to define categorical variables. Apart from these, in comparison of other variables, Student's t-test or Mann-Whitney test was used. Spearman and Pearson tests were used in the correlation analysis according to the distribution characteristics of the data. $\mathrm{P}<0.05$ value was accepted as statistically significant.

\section{Results}

The study included a total of 103 NAFLD patients. Fiftyfive patients had metabolic syndrome (MS) while 48 did not have. The control group included 50 healthy volunteers who were in same age and gender range. Alcohol use, hepatotoxic drug use, hepatitis markers, HIV Ab and autoimmune markers were negative in all groups. Demographic data of all patients and healthy volunteers are given as a table. (Table 1). 
Table 1. Comparison of demographic characteristics of patients

\begin{tabular}{|c|c|c|c|c|}
\hline & Metabolic syndrome (-) & Metabolic syndrome (+) & Control group & $\mathrm{p}$ \\
\hline Age(years) & $47.7 \pm 10.57$ & $49.98 \pm 9.77$ & $45.86 \pm 10.08$ & 0.072 \\
\hline $\mathrm{BMI}(\mathrm{kg} / \mathrm{m} 2)$ & $29.44 \pm 3.47$ & $32.2 \pm 4.31$ & $24.5 \pm 2.88$ & 0.001 \\
\hline $\mathrm{WC}(\mathrm{cm})$ & $97.05 \pm 8.07$ & $103.57 \pm 10.51$ & $78.2 \pm 8.5$ & 0.001 \\
\hline WC/HC & $0.9 \pm 0.07$ & $0.93 \pm 0.074$ & $0.78 \pm 0.08$ & 0.001 \\
\hline \multicolumn{5}{|l|}{ Gender } \\
\hline Male & $22(\% 45.8)$ & $17(\% 30.9)$ & $15(\% 30)$ & 0.182 \\
\hline Female & $26(\% 54.2)$ & $38(\% 69.1)$ & $35(\% 70)$ & \\
\hline \multicolumn{5}{|l|}{ HTN } \\
\hline Present & $5(\% 10.4)$ & $24(\% 43.6)$ & 0 & 0.001 \\
\hline Absent & $43(\% 89.6)$ & $31(\% 56.4)$ & 50 & \\
\hline \multicolumn{5}{|l|}{$\mathrm{DM}$} \\
\hline Present & $4(8.3)$ & $24(\% 43.6)$ & 0 & 0.001 \\
\hline Absent & $44(91.7)$ & $31(\% 56.4)$ & 50 & \\
\hline \multicolumn{5}{|l|}{ Smoking } \\
\hline Present & $7(\% 14.6)$ & $5(\% 9.1)$ & $5(\% 10)$ & 0.023 \\
\hline Absent & $41(\% 85.4)$ & $50(\% 90.9)$ & $45(\% 90)$ & \\
\hline \multicolumn{5}{|l|}{ Alcohol use } \\
\hline Present & 0 & 0 & 0 & - \\
\hline Absent & $48(\% 100)$ & $55(\% 100)$ & $50(\% 100)$ & \\
\hline \multicolumn{5}{|l|}{ Drug use } \\
\hline Present & 0 & 0 & 0 & - \\
\hline Absent & $48(\% 100)$ & $55(\% 100)$ & $50(\% 100)$ & \\
\hline \multicolumn{5}{|c|}{ Hepatitis marker } \\
\hline$(+)$ & 0 & 0 & 0 & - \\
\hline$(-)$ & $48(\% 100)$ & $55(\% 100)$ & $50(\% 100)$ & \\
\hline \multicolumn{5}{|c|}{ Autoimmune marker } \\
\hline$(+)$ & 0 & 0 & 0 & - \\
\hline$(-)$ & $48(\% 100)$ & $55(\% 100)$ & $50(\% 100)$ & \\
\hline
\end{tabular}

BMI(body mass index), DM (diabetes mellitus), HC(hip circumference), HTN(hypertension) WC(waist circumference), HC(hip circumference).

We evaluated the ultrasonographic stages of fatty liver and presence of hepatomegaly in all groups. And there were 37 patients with grade II, 7 patients with grade II-III, 4 patients with grade III hepatosteatosis in the group without MS while there were 32 patients with grade II, 13 patients with grade II-III and 10 patients with grade III hepatosteatosis in the group with MS. When we analyzed both of these groups, in terms of their ultrasonographic stages of hepatosteatosis, we couldn't find any statistically significantly difference between them. There were 19 patients with hepatomegaly in the group without MS and 39 patients with hepatomegaly in the group with MS.

When we analyzed laboratory parameters (ALT, AST, GGT, glucose, IR, HDL, TG, d.LDL, hs-CRP, HbA1c) for study population, all laboratory parameters except HDL were statistically significantly higher in NAFLD patients than they were in control group. Fasting glucose, TG and d.LDL levels were also higher in NAFLD patients with MS than they were in NAFLD patients without MS (Table 2).
Maximum and mean c-imt and carotid cross sectional area values were higher in all patients than they were in healthy volunteers. But when we compared the NAFLD groups, there was no statistically significant difference for these parameters in patients with MS and without MS. We found carotid artery plaques in 11 patients in the group with MS, and 3 patients in the group without MS, and the difference was statistically significant (Table 2). 
Table 2. Comparison of laboratory parameters and carotid intima-media thickness and carotid cross sectional area levels in groups

\begin{tabular}{|l|l|l|l|l|l|}
\hline & NAFLD with MS & $\begin{array}{l}\text { NAFLD without } \\
\text { MS }\end{array}$ & Control & p & $\begin{array}{l}\text { NAFLD vs Control } \\
\text { P }<0.017\end{array}$ \\
\hline Avg. c-IMT max $(\mathrm{cm})$ & $0.869 \pm 0.07$ & $0.831 \pm 0.07$ & $0.724 \pm 0.05$ & $<0.001$ & $\beta, \alpha$ \\
\hline Avg. c-IMT mean $(\mathrm{cm})$ & $0.733 \pm 0.06$ & $0.713 \pm 0.07$ & $0.619 \pm 0.04$ & $\leq 0.001$ & $\beta, \alpha$ \\
\hline Avg. c-CSA $\left(\mathrm{cm}^{2}\right)$ & $21.487 \pm 2.16$ & $21.014 \pm 2.24$ & $18.450 \pm 1.41$ & $<0.001$ & $\beta, \alpha$ \\
\hline Hs CRP $(\mathrm{mcg} / \mathrm{dL})$ & $0.47 \pm 0.5$ & $0.36 \pm 0.7$ & $0.11 \pm 0.1$ & $=0.003$ & $\beta$ \\
\hline HOMA-IR & $4.36 \pm 2.2$ & $2.61 \pm 1.3$ & $1.30 \pm 0.5$ & $\leq 0.001$ & $\beta, \alpha, \#$ \\
\hline ALT(U/L) & $44.09 \pm 23.3$ & $41.85 \pm 30.9$ & $15.76 \pm 5.0$ & $<0.001$ & $\alpha, \beta$ \\
\hline AST $(\mathrm{U} / \mathrm{L})$ & $32.60 \pm 15.1$ & $29.75 \pm 13.1$ & $18.74 \pm 4.3$ & $<0.001$ & $\alpha, \beta$ \\
\hline GGT $(\mathrm{U} / \mathrm{L})$ & $41.87 \pm 37.8$ & $34.10 \pm 28.7$ & $17.84 \pm 8.9$ & $<0.001$ & $\alpha, \beta$ \\
\hline ALP(U/L) & $204.56 \pm 62.4$ & $186.39 \pm 49.4$ & $141.12 \pm 40.2$ & $<0.001$ & $\alpha, \beta$ \\
\hline Glucose $(\mathrm{mg} / \mathrm{dl})(\mathrm{mg} / \mathrm{dL})$ & $106.21 \pm 24.0$ & $90.70 \pm 14.9$ & $81.10 \pm 6.8$ & $<0.001$ & $\alpha, \beta, \#$ \\
\hline HBA1C $(\mathrm{mmol} / \mathrm{mol})$ & $6.17 \pm 0.8$ & $5.56 \pm 0.5$ & $5.43 \pm 0.3$ & $<0.001$ & $\beta, \#$ \\
\hline HDL $(\mathrm{mg} / \mathrm{dL})$ & $43.12 \pm 9.5$ & $51.37 \pm 12.3$ & $59.42 \pm 11.4$ & $<0.001$ & $\alpha, \beta, \#$ \\
\hline TG $(\mathrm{mg} / \mathrm{dL})$ & $176.01 \pm 81.7$ & $132.62 \pm 56.8$ & $76.18 \pm 25.8$ & $<0.001$ & $\alpha, \beta, \#$ \\
\hline dLDL $(\mathrm{mg} / \mathrm{dL})$ & $121.05 \pm 37.4$ & $139.87 \pm 36.7$ & $92.91 \pm 22.3$ & $<0.001$ & $\alpha, \beta$ \\
\hline
\end{tabular}

$\alpha$ : NAFLD without metabolic syndrome versus control

B: NAFLD with metabolic syndrome versus control

\#: NAFLD with metabolic syndrome versus NAFLD without metabolic syndrome

ALT(alanine aminotransferase), ALP(alkaline phosphatise), AST (aspartate aminotransferase), Avg(average), c-CSA(carotid cross sectional area), c-imt(carotid intima media thickness), d-LDL(direct low density lipoprotein), GGT(gamma-glutamyl transpeptidase), HDL(high density lipoprotein), HOMA-IR(homa-insulin resistance) Hs-CRP (high sensitive C reactive protein), TG(triglyceride)

When we analyzed HOMA-IR values for the study population, we found higher HOMA-IR levels in patients than they were in control group. We compared HOMA-IR levels in patients presence of MS and in the group with MS we found HOMA-IR levels higher than in the group without MS. The number of HOMA-IR positive patients in the group with MS were 47 and the number of HOMA-IR negative patients in the same group were 8 . And there were 21 HOMA-IR positive patients and 27 HOMA-IR negative patients in the group without MS. When we compared these HOMA-IR positive and negative patients in the group without MS, according to the c-imt values, there was no statistically significant difference between them. So based on these results, in NAFLD patients without metabolic syndrome, insulin resistance does not play a role in higher c-imt values compared to the control group (table 2).

Hs-CRP values were significantly higher in patients compared to the control group. But in the subgroup analyses there was no difference between NAFLD without MS patients and control group.

Finally, correlation-regression analysis was performed for every laboratory parameter which could affect carotid intima media thickness. Only hs-CRP values showed a low correlation with carotid intima media thickness in the group with MS but in the group without MS, there was no positive or negative correlation between hs-CRP and c-imt values. There was no correlation between ALT, GGT, HDL, TG, d.LDL values, and c-imt values in both the MS-positive and negative groups.

\section{Discussion}

The incidences of nonalcoholic fatty liver disease in adults and children are rapidly increasing due to ongoing epidemics of obesity and type 2 diabetes in Western and recently in Asian populations. ${ }^{14,15}$ There are few increasing reports that suggest a relationship between NAFLD and the risk of increased cardiovascular morbidity. ${ }^{16}$ The relationship between cardiovascular risk factors and NAFLD can explain the high risk of cardiovascular disease in patients with fatty liver. ${ }^{16,17}$ Recent case-control and regional studies showed increased carotid intima- media thickness in patients with NAFLD. ${ }^{18-21}$ Moreover, ample evidence associates NAFLD with endothelial dysfunction, increased pulse wave velocity and increased coronary arterial calcifications all of which are established markers for cardiovascular disease. ${ }^{22}$

In this study, we demonstrated that patients with NAFLD had evidence of increased carotid intima media thickness compared to healthy controls.

Endothelial dysfunction, oxidative stress, inflammation, inflammatory cytokines, and abnormal lipid and glucose metabolism are suggested as possible biological mechanisms of atherosclerosis in NAFLD. ${ }^{16}$ On the other hand, these mechanisms are also related to other metabolic risk factors. Consequently, it is not clear whether NAFLD has a direct effect on the development of atherosclerosis. In some prospective cohort studies, it is showed that coronary heart disease is associated with NAFLD and this relationship is independent of 
classic risk factors for coronary heart disease. ${ }^{22,23}$ This suggest epidemiological evidence for NAFLD promoting cardiovascular heart disease. In our study, one of the main aims was to find out if NAFLD is an independent risk factor for atherosclerosis.

In a meta-analysis of Sookoian and Pirola containing 3497 people from 7 separate studies, a relationship was found between NAFLD and carotid intima- media thickness. The degree of carotid intima-media thickness in patients with fatty liver was $13 \%$ higher than people without fatty liver. Also, there was a strong correlation between fatty liver and at least one plaque formation. At the same time, the prevalence of elevated liver function tests, carotid intima-media thickness and the presence of plaque was significantly related. According to researchers of the study, the power of this study was extensive in this patient population and it is the first study that offers evidence-based data. ${ }^{4}$ In a recent meta-analysis of seven studies on the relationship between NAFLD and carotid intima-media thickness, it is showed that NAFLD can promote the increase of intima-media thickness and the increased carotid intima-media thickness has a strong relationship with the severity of NAFLD.In addition; Cai et al. reported that the risk of carotid plaque in NAFLD patients was 3.73 times of that in non NAFLD patients. ${ }^{24}$ In a study by Kim et al., there was a strong relationship between NAFLD and coronary artery calcification, the latter being established surrogate marker of coronary atherosclerosis and authors reported that this association was independent of the traditional risk factors. ${ }^{25}$

In our study, carotid intimal and medial thickness was higher in all NAFLD patients compared to the control group regardless of the presence of metabolic syndrome. Carotid intimal and medial thickness was significantly higher in the NAFLD patients with MS compared to the patients without MS. These values were also higher in the group that NAFLD without MS compared to the control group.

In the study by Targher and Arcaro, there is evidence suggesting that NAFLD has a relationship with increased cardiovascular mortality and major cardiac adverse events independently. ${ }^{16}$ Their data supports the idea that NAFLD and atherosclerosis may have a common molecular mediator with atherosclerosis, and that NAFLD may not be just a risk factor for cardiovascular disease but also an early mediator for atherosclerosis. A recent observational study conducted at the same center in patients with biopsy-supported NAFLD showed that these patients had higher carotid intima media thickness compared to the control group. ${ }^{26}$ At the same time, carotid intima media thickness was higher in patients with NAFLD compared to patients with simple steatosis. As the severity of NAFLD increases histologically, c-imt increase independent of classic risk factors, HOMA-IR, and metabolic syndrome components.

The relationship between NAFLD and metabolic disease, and c-imt has been previously studied. The concurrent presence of these two diseases in a patient increases the risk of non-alcoholic steatohepatitis. The presence of metabolic syndrome in patients with NAFLD was related to non-alcoholic steatohepatitis, and more severe necro-inflammatory activity and fibrosis. ${ }^{27}$

In our study, NAFLD was diagnosed via B-mode ultrasonography and by eliminating other causes of fatty liver, however, there was no histopathological evaluation. When we compared ultrasonographic grades of the MS-negative and positive groups, there was no statistically significant difference.
However, as the ultrasonographic grade of hepatosteatosis increases, c-imt also increased.

Ahmed et al. reported that NAFLD stimulates insulin resistance and leads accelerated atherosclerosis. ${ }^{28} \mathrm{~A}$ previous study demonstrated that there was a strong relationship between hepatic fat content and hepatic insulin sensivity. ${ }^{29}$ Kwak et al. suggested that insulin resistance could be amplified in NAFLD patients with hyperglycemia, which may be related to the presence of coronary atherosclerosis. Also authors reported that there was a link between oxidative stress, inflammation and NAFLD, hyperglycemia, insulin resistance and cardiovascular disease. ${ }^{30}$ Hepatocyte injury induced by hepatic steatosis causes cytokines releasing and this provides pro-inflammatory status which progresses the liver damage and adds further atherogenic stimuli.

In our study, 47 (85.5\%) of NAFLD patients with MS were positive for HOMA-IR. In this group, carotid intima-media thickness were higher compared to the NAFLD without MS group and the control group. When we compared the patients in NAFLD without MS group between the HOMA-IR positive $(\mathrm{n}=21, \% 43.8)$ and negative groups $(\mathrm{n}=27, \% 56.3)$, there was no significant difference in carotid intima-media thickness. This situation suggests that insulin resistance has no role in increased carotid intima media thickness in the group that NAFLD without MS.

In a study by Sookoian and Pirola, a relationship between liver enzymes and carotid atherosclerosis was found, and this was most notable between carotid intima-media thickness, and ALT and $\gamma$-GGT values. These findings are consistent with epidemiological studies, reporting a relationship between increased serum liver enzyme levels, atherothrombotic risk profile, and increased cardiovascular disease risk. ${ }^{3}$ However, in our study, ALT and GGT levels were higher in NAFLD patients compared to the control group, while there was no correlation between ALT and GGT values and carotid intima-media thickness. There was no difference between with or without MS groups in terms of ALT, AST, and GGT values .

In a study conducted in young, non-obese Asian patients, NAFLD was related to hypertension, diabetes, high CRP levels, metabolic syndrome, and an increased Framingham risk score. This study was a wide-ranging study that analyzed the relationship between hepatic steatosis and several cardiovascular risk factors in a healthy group of people. In this study, an increase in hs-CRP levels was shown in the NAFLD and nonalcoholic steatohepatitis (NASH) groups. ${ }^{31}$ In many prospective studies, hs-CRP has been shown to be a precursor of myocardial infarction. High CRP levels are related to other characteristics of metabolic syndrome even in healthy individuals. Increased insulin resistance and atherogenic dyslipidemia along with proatherogenic factors like C-reactive protein released from the liver may lead to progression of cardiovascular disease. ${ }^{32}$

In our study, hs-CRP levels were higher in the group with MS (min:0.04 max.:3.2 median:0.29) and the group without MS (min:0.02 max.:4.8 median:0.17) compared to the control group (min:0.01 max.:0.66 median:0.08). Hs-CRP levels were higher in the group with MS compared to the group without MS. However there was a slightly positive correlation between hs-CRP levels and c-imt values only in the group with MS. There was no positive or negative correlation between hs-CRP and c-imt values in the group without MS. 
As a result, carotid intima-media thickness was higher in all NAFLD patients which are with or without metabolic syndrome compared to the control group. In patients with NAFLD without metabolic syndrome, we could not find a significant difference between patients with positive and negative insulin resistance, thus increased thickness of carotid intima-media could not be explained by the presence of metabolic syndrome. From this point of view, one can say that our study supports the relationship between NAFLD and cardiovascular disease. The risk of NAFLD has an independent relationship to insulin resistance and other metabolic risk factors. This risk is increased as other metabolic risk factors are concomitant to NAFLD.

\section{Study Limitations}

The relatively low number of patients, age and gender matchup of the control group, and not having a histopathological diagnosis of NAFLD and oxidative stress indicators that can be related to an increased risk of cardiovascular disease are the limitations of this study. More studies are required to show the relationship between NAFLD and atherosclerosis, and to determine whether it is an independent risk factor for cardiovascular disease.

\section{Conclusion}

In conclusion, NAFLD was independently associated with subclinical carotid atherosclerosis. In NAFLD patients, routine carotid ultrasonography examination could help to predict carotid atherosclerosis occurrence and assess their risk of cardiovascular disease.

\section{References}

1. Bellentani S, Scaglioni F, Marino M, et al. Epidemiology of non-alcoholic fatty liver disease. Dig Dis 2010; 28: 155-61. https://doi.org/10.1159/000282080

2. Gupte P, Amarapurkar D, Agal S, et al. Non-alcoholic steatohepatitis in type 2 diabetes mellitus. J Gastroenterol Hepatol. 2004;19: 854-858. https://doi.org/10.1111/j.1440-1746.2004.03312.x

3. Sookoian S, Pirola CJ. Non-alcoholic fatty liver disease is strongly associated with carotid atherosclerosis: a systematic review. J Hepatol. 2008;49:600-607 https://doi.org/10.1016/j.jhep.2008.06.012

4. Rhee EJ, Lee WY, Cho YK, et al. Hyperinsulinemia and the development of nonalcoholic Fatty liver disease in nondiabetic adults. Am J Med. 2011;124:69-76. https://doi.org/10.1016/j.amjmed.2010.08.012

5. Adams LA, Angulo P. Recent concepts in non-alcoholic fatty liver disease. Diabet Med 2005; 20: 1129-33. https://doi.org/10.1111/j.1464-5491.2005.01748.x

6. McCullough AJ. Pathophysiology of non-alcoholic steatohepatitis. J Clin Gastroenterol 2006;40 : 17-29

7. Treeprasertsuk S, Lopez-Jimenez F, Lindor KD. Nonalcoholic fatty liver disease and the coronary artery disease. Dig Dis Sci 2011; 56: 35-45.

https://doi.org/10.1007/s10620-010-1241-2

8. De Groot E, Hovingh GK, Wiegman A, et al. Measurement of arterial wall thickness as a surrogate marker for atherosclerosis. Circulation. 2004 Jun 15;109(23 Suppl 1):III33-8. https://doi.org/10.1161/01.CIR.0000131516.65699.ba

9. Zureik M1, Ducimetière P, Touboul PJ, et al. Common carotid intima-media thickness predicts occurrence of carotid atherosclerotic plaques: longitudinal results from the Aging Vascular Study (EVA) study. Arterioscler Thromb Vasc Biol. 2000 Jun;20(6):1622-9.

https://doi.org/10.1161/01.ATV.20.6.1622
10. Carnicelli AP, Stone JJ, Doyle A, et al. Cross-sectional area for the calculation of carotid artery stenosis on computed tomographic angiography. J Vasc Surg. 2013;58(3):659665. https://doi.org/10.1016/j.jvs.2013.02.031

11. Eigenbrodt ML, Bursac Z, Tracy RE, et al. B-mode ultrasound common carotid artery intima-media thickness and external diameter: Cross-sectional and longitudinal associations with carotid atherosclerotic in a large population sample. Cardiovasc Ultrasound 2008; 6: 1-11. https://doi.org/10.1186/1476-7120-6-10

12. World Health Organization. Definition, diagnosis and classification of diabetes mellitus and its complications: report of a WHO Consultation. Part 1: diagnosis and classification of diabetes mellitus. Geneva, Switzerland: World Health Organization; 1999. Available at: http:// whqlibdoc.who.int/hq/1999/WHO_NCD_NCS_99.2.pdf. Accessed December 12, 2003.

13. Matthews DR, Hosker JP, Rudenski AS, et al. Homeostasis model assessment: insulin resistance and $\beta$-cell function from fasting plasma glucose and insulin concentrations in man. Diabetologia.1985;28:412- 419 https://doi.org/10.1007/BF00280883

14. Lee JY, Kim KM, Lee SG, et al. Prevalence and risk factors of non-alcoholic fatty liver disease in potential living liver donors in Korea: a review of 589 consecutive liver biopsies in a single center. J Hepatol. 2007;47:239-244. https://doi.org/10.1016/j.jhep.2007.02.007

15. Tsuneto A, Hida A, Sera N, et al. Fatty liver incidence and predictive variables. Hypertens Res. 2010;33:638-643. https://doi.org/10.1038/hr.2010.45

16. Targher G, Arcaro G. Non-alcoholic fatty liver disease and increased risk of cardiovascular disease. Atherosclerosis 2007; 191: 235-40. https://doi.org/10.1016/j.atherosclerosis.2006.08.021

17. Kim HC, Choi SH, Shin HW, et al. Severity of ultrasonographic liver steatosis and metabolic syndrome in Korean men and women. World J Gastroenterol 2005; 11: 5314-21. https://doi.org/10.3748/wjg.v11.i34.5314

18. Fracanzani AL, Burdick L, Raselli S, et al. Carotid artery intima media thickness in nonalcoholic fatty liver disease. Am J Med 2008; 121: 72-8.

https://doi.org/10.1016/j.amjmed.2007.08.041

19. Targher G, Bertolini L, Padovani R, et al. Non-alcoholic fatty liver disease is associated with carotid artery wall thickness in diet-controlled type 2 diabetic patients. J Endocrinol Invest 2006; 29: 55-60. https://doi.org/10.1007/BF03349177

20. Targher G, Bertolini L, Padovani R, et al. Relations between carotis artery wall thickness and liver histology in subjects with nonalcoholic fatty liver disease. Diabetes Care 2006; 29: 1325-30. https://doi.org/10.2337/dc06-0135

21. Choi SY, Kim D, Kang JH, et al. Nonalcoholic fatty liver disease as a risk factor of cardiovascular disease: relation of nonalcoholic fatty liver disease to carotid atherosclerosis. Korean J Hepatol 2008; 14: 77-88.

https://doi.org/10.3350/kjhep.2008.14.1.77

22. Francque SM, van der Graaff D, Kwanten WJ. Nonalcoholic fatty liver disease and cardiovascular risk: pathophysiological mechanisms and implications. J Hepatol. 2016 Apr 15. https://doi.org/10.1016/j.jhep.2016.04.005

23. Elsheikh E, Younoszai Z, Otgonsuren M, et al. Markers of endorthelial dysfunction in patients with nonalco $\neg$ holic fatty liver disease and coronary artery disease. J Gastroenterol Hepatol 2014; 29: 1528-34. https://doi.org/10.1111/jgh.12549 
24. Cai J, Zhang S, Huang W. Association between non alcoholic fatty liver disease and carotid atherosclerosis : a meta-analysis. Int J Clin Exp Med 2015 ; 8: 7673-8.

25. Kim D, Choi SY, Park EH, et al. Nonalcoholic fatty liver disease is associated with coronary artery calcification. Hepatology 2012;56:605-13. https://doi.org/10.1002/hep.25593

26. Targher G, Bertolini L, Padovani R, et al. Relation of nonalcoholic hepatic steatosis to early carotid atherosclerosis in healthy men: role of visceral fat accumulation. Diabetes Care 2004; 27: 2498-500.

https://doi.org/10.2337/diacare.27.10.2498

27. Marchesini G, Bugianesi E, Forlani G, et al. Nonalcoholic fatty liver, steatohepatitis and the metabolic syndrome. Hepatology 2003; 37: 917-23. https://doi.org/10.1053/jhep.2003.50161

28. Ahmed MH, Barakat S, Almobarak AO. Nonalcoholic fatty liver disease and cardiovascular disease: has the time come for cardiologists to be hepatologists? J Obes 2012;2012:483135.

https://doi.org/10.1155/2012/483135

29. Yki-Jarvinen H, Westerbacka J. The fatty liver and insulin resistance. Curr Mol Med 2005;5:287-95. https://doi.org/10.2174/1566524053766031

30. Kwak MS, Yim YJ, Kim D, et al. Non alcoholic fatty liver disease is associated with coronary artery calcium score in diabetes patients with higher HbAlc. Diabetology\& Metabolic Syndrome 2015, 7:28 doi:10.1186/s13098-0150025-4 https://doi.org/10.1186/s13098-015-0025-4

31. Sung KC, Ryan MC, Wilson AM. The severity of nonalcoholic fatty liver disease is associated with increased caridovascular risk in a large cohort of non-obese Asian subjects. Atherosclerosis 2009; 203: 581-6. https://doi.org/10.1016/j.atherosclerosis.2008.07.024

32. Non-Alcoholic Fatty Liver Disease: a Clinical Spectrum. by Gourdas Choudhuri, Elsevier, 2013:6-9. 